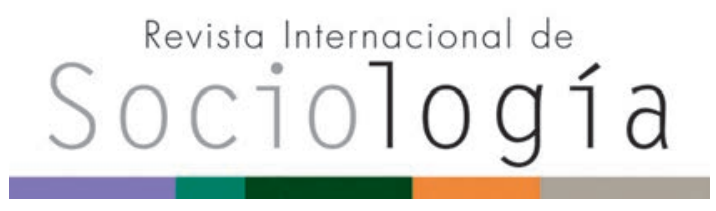

Revista Internacional de Sociología RIS

vol. 77 (3), e131, julio-septiembre, 2019, ISSN-L:0034-9712 https://doi.org/10.3989/ris.2019.77.3.18.027

\section{LA DISTRIBUCIÓN DEL TIEMPO EN LOS HOGARES MONOPARENTALES DE MADRE OCUPADA. VIVIR CON OTROS COMO ESTRATEGIA DE CONCILIACIÓN}

\author{
MARC AJENJo Cosp \\ Centre d'Estudis Demogràfics. Universitat Autònoma de Barcelona \\ majenjo@ced.uab.cat \\ ORCID iD: https://orcid.org/0000-0002-6912-6264 \\ NúRIA GARCÍA SALADRIGAS \\ Centre d'Estudis Demogràfics. Universitat Autònoma de Barcelona \\ ngarcia@ced.uab.cat \\ ORCID iD: https://orcid.org/0000-0002-8711-6836
}

Cómo citar este artículo / Citation: Ajenjo Cosp, M. y N. García Saladrigas. 2019. "La distribución del tiempo en los hogares monoparentales de madre ocupada. Vivir con otros como estrategia de conciliación". Revista Internacional de Sociología 77(3):e131. https://doi. org/10.3989/ris.2019.77.3.18.027

\section{Resumen}

Este artículo analiza la distribución del tiempo destinado al trabajo no remunerado — cuidado y tareas domésticas- en los hogares monoparentales de madre ocupada a partir de la Encuesta de Empleo del Tiempo 2009-10. Se parte de la hipótesis de que la madre monoparental ocupada lleva a cabo dos estrategias de conciliación: reorganizar su tiempo y convivir con otros familiares ajenos al núcleo. Los resultados muestran que estas madres dedican el mismo tiempo al cuidado que las biparentales ocupadas, mientras que el tiempo dedicado a tareas del hogar es menor, especialmente en aquellas actividades más arduas, como cocinar o limpiar. Por otro lado, las madres monoparentales ocupadas viven con miembros ajenos al núcleo en una proporción muy superior sus homólogas biparentales. La aportación de estos miembros ajenos al núcleo al trabajo de cuidado es significativamente superior en los hogares monoparentales, algo que no se ha podido demostrar para el trabajo doméstico.

\section{Palabras Clave}

Hogares monoparentales; Madres ocupadas; Tareas del hogar; Tiempo de cuidado; Uso del tiempo.
Copyright: () 2019 CSIC. Este es un artículo de acceso abierto distribuido bajo los términos de la licencia de uso y distribución Creative Commons Reconocimiento 4.0 Internacional (CC BY 4.0).

Recibido: 16/02/2018. Aceptado: 17/01/2019. Publicado online: 24/09/2019

\section{ABSTRACT}

This paper analyses single-mother households' unpaid work distribution -childcare and houseworkcompared with dual-parent households using the EET 2009-10. We hypothesize that working single-mothers implement two balance strategies: rearrange their time and live with rela-tives that are not part of the nuclear family. Results show that while working single-mothers invest the same amount of time in childcare as dual-parent mothers, the time in-vested in housework is lower, especially in time-demanding activities such as cooking or cleaning. On the other hand, single-mothers more often live with other people compared to dual-parent mothers. Furthermore, other members' time contribution to childcare is significantly higher in single-mother households, but this tendency is not observed for housework.

\section{KeYWORDS}

Care; Housework; Single-mother households; Time use; Working mothers. 


\section{INTRODUCCIÓN}

El número de hogares formados por un solo progenitor con hijos no ha dejado de aumentar en España desde las últimas décadas del siglo pasado (Becerril, 2004). El incremento de rupturas matrimoniales (Fernández y Tobío, 1998; Meil, 1999) y de las separaciones de las parejas de hecho (Dominguez-Folgueras, 2011), así como la reciente proliferación de las madres solteras por elección (Avilés y Sánchez, 2015), son los principales factores que explican esta expansión. En este contexto, la monoparentalidad femenina sigue siendo claramente mayoritaria (Avilés, 2015).

En otro orden de cosas, las mujeres se enfrentan a la sobrecarga de actividades y a los problemas de conciliación entre vida laboral y familiar. La "doble jornada" (Hochschild y Machung, 1989; Durán, 1986) o "doble presencia-ausencia" (Izquierdo, 1998) afecta cada día a un mayor número de mujeres. Algunas autoras identifican, incluso, una "triple presencia-ausencia", añadiendo a las responsabilidades laborales y del hogar aquellas relacionadas con la gestión y planificación en el ámbito doméstico (Sagastizabal y Legarreta, 2016). Esta desigualdad en el ámbito familiar se contrapone a la mayor equidad en la esfera laboral y educativa. La revolución igualitaria evoluciona lentamente por lo que algunos autores apuntan a su estancamiento (EspingAndersen, 2009; England, 2010), originando la llamada "incompleta revolución de género" (Goldscheider, 2000; McDonald, 2000). Más recientemente, (Goldscheider, Bernhardt y Lappegard, 2015) apuntan que, a pesar de las múltiples diferencias tanto regionales como entre clases sociales, la mayor implicación del hombre en el trabajo no remunerado sigue impulsando la revolución de género.

En este sentido, algunos estudios han señalado una dinámica esperanzadora en parejas que podríamos denominar menos tradicionales. Las parejas de doble ingreso (Infestas, 2015; GarcíaRomán, 2012), las cohabitantes (Ajenjo y GarcíaRomán, 2011; González y Jurado, 2009) y las reconstituidas (Treviño, Gumà y Permanyer, 2013) tienen una distribución del trabajo reproductivo más equitativa que las parejas tradicionales. A pesar de ello, y de la emergencia del llamado "nuevo padre" (González y Jurado 2015; Gracia, 2014), el cual participa activamente en el cuidado de los hijos, la mayor contribución de los hombres a las tareas de cuidado no se ha traducido en una disminución del tiempo de la mujer (Moreno, Ajenjo y Borràs, 2016; Domínguez-Folgueras, 2015; Bianchi, 2011), ya que estas tareas, a diferencia de otras - como las tareas domésticas-, parecen no ser substituibles.

En este contexto de mayor carga de trabajo para la mujer, el presente artículo identifica dos de las estrategias de conciliación seguidas por las madres monoparentales españolas: la redistribución de su tiempo y la convivencia con otras personas. Primero, se examina la distribución del tiempo que las madres monoparentales dedican al trabajo no remunerado, dividido en cuidado y tareas del hogar, comparándolo con el tiempo de las madres biparentales. Después, se analiza el uso del tiempo del resto de integrantes del hogar: los hijos e hijas, así como los miembros del hogar ajenos al núcleo, centrando el foco de atención en valorar hasta qué punto su presencia es un factor clave para la conciliación.

\section{Antecedentes}

A pesar de tener un buen número de características en común, la monoparentalidad engloba heterogeneidad de perfiles, orígenes, circunstancias vitales y situaciones familiares. Una muestra de ello es la diversidad de rutas de entrada a la monoparentalidad, siendo la más frecuente, a día de hoy, la ruptura de una unión con hijos. Si bien la monoparentalidad había estado tradicionalmente vinculada a esta ruptura, ya sea por defunción o separación, las nuevas técnicas de reproducción asistida, junto con la adopción por parte de mujeres solas, permiten un tipo claramente diferenciado, las "madres solteras por elección" (MSPE) (Avilés y Sánchez, 2015; Rivas y Jociles, 2013). ${ }^{1}$

Aunque en las últimas décadas los datos apuntaban a una mayor presencia en el mercado laboral por parte de las madres monoparentales, los datos más recientes indican cierta equiparación. Para la mayor parte de las madres monoparentales, al no disponer de otra fuente de ingresos, el trabajo remunerado es ineludible. En algunos casos, el divorcio o separación explican directamente la vuelta al mercado de trabajo mientras que, en otros, el trabajo remunerado formaba parte de una estrategia de autonomía personal e independencia anterior a la ruptura (Fernández y Tobío, 1999). Para las MSPE, la autonomía económica es precisamente una condición previa a la monoparentalidad (González et al., 2008). Más allá de la especificidad de este colectivo, muchos autores han identificado una mayor propensión a la pobreza y a la exclusión social de las familias monoparentales (Almeda y Di Nella, 2012; González, Jiménez y Morgado, 2004). Según datos de la OCDE, el $39 \%$ de las familias monoparentales en España se hallan en riesgo de pobreza (Castro, 2015).

Las madres monoparentales se enfrentan a mayor precariedad laboral, viéndose forzadas a aceptar trabajos por debajo de su perfil formativo y capacidad laboral y con largas jornadas laborales (Almeda, Di Nella y Obiol, 2008). La conciliación es uno de los mayores obstáculos con el que se encuentran, enfrentándose a la escasez de tiempo y la sobrecarga de actividades. Hacer compatibles los horarios laborales con los infantiles es un problema generalizado entre las mujeres trabajadoras, pero, si a este hecho se le añade la situación de monoparentalidad, el problema se ve agravado. 
Son diversas las estrategias a las que las madres monoparentales recurren para poder compatibilizar la vida laboral y familiar. Una de las más habituales es pedir ayuda a la familia extensa, siendo especialmente importante la figura de la abuela, quien responde a las necesidades del cuidado informal (Seiz, 2016; Hernández-Monleón, 2016; Jiménez, 2003). Otra de las estrategias utilizadas es la de minimizar desplazamientos buscando la proximidad espacial de las actividades cotidianas - casa, trabajo y escuela-, a las que frecuentemente se añade la casa de los abuelos (Fernández y Tobío, 1999). Y es que cuanto más próxima esté una persona, geográfica o afectivamente, más probable es que preste ayuda (Meil, 2011). De hecho, la co-residencia ha sido identificada como una forma de solidaridad familiar (Albertini, Kohli y Vogel, 2007) que permite paliar las dificultades económicas y sociales de las madres monoparentales (Treviño, 2011).

Otra pieza esencial para comprender cómo se desarrolla la cotidianeidad en esta tipología de hogar es el papel que juegan los descendientes. Aunque algunos estudios concluyen que los hijos de familias monoparentales participan más de las tareas domésticas que los hijos de familias con dos progenitores (Gager, Cooney y Call, 1999; Demo y Acock, 1993; Goldscheider y Waite, 1991), en otros más recientes se identifica la mayor participación únicamente bajo el régimen de custodia compartida de los padres (Gager, Sanchez y Demaris, 2009). La implicación de los hijos en las tareas del hogar aumenta con la edad (Maganto, Bartau y Etxebarría, 2003) y los hijos monoparentales adquieren responsabilidades a edades más tempranas (Longfellow, 1979). En dichas edades ya existen diferencias de género, las hijas participan más de las tareas del hogar (Ajenjo y García-Román, 2014; Gager, Sanchez y Demaris, 2009; Bianchi y Robinson, 1997; Blair, 1992) y realizan tareas más "feminizadas" (p. ej., cocinar o limpiar) dentro del mismo (Meil, 2008; Evertsson, 2006; Raley, 2006). Estas diferencias se incrementan durante los fines de semana (Ajenjo y García-Román, 2014) cuando la aportación es mayor (Sayer, Gauthier y Fustenberg, 2004) y, por ende, más desigual.

En este punto es importante retomar las diferencias existentes dentro del colectivo de madres monoparentales, en el sentido de que la existencia o no de la figura del progenitor no residente puede generar grandes diferencias. Lejos queda la figura del padre "ausente" (Brullet et al., 2011), siendo cada vez más habitual que ambos progenitores compartan no solo los cuidados, sino también la custodia de su progenie (Solsona, Brullet y Spijker, 2014). En este sentido, la existencia de un padre que invierta no solo dinero, sino también tiempo en la crianza de los hijos, tiene un impacto directo en las estrategias de conciliación de las madres monoparentales. Precisamente, un estudio reciente muestra que los niños que viven en un hogar monoparental comparten más tiempo con miembros ajenos al hogar (Nazio, 2017). Desafortunadamente, estos parámetros no van a poder ser analizados en este artículo, ya que las encuestas de las que disponemos recogen información exclusivamente del hogar, dejando fuera aquellos familiares que no residen entre sus muros.

Existen dos teorías especialmente relevantes para comprender la participación de la mujer en el trabajo no remunerado. ${ }^{2}$ La primera de ellas, la perspectiva de la disponibilidad de tiempo, asume que el tiempo y las energías humanas son finitas (Marks, 1977; Coser, 1974), dado que el tiempo dedicado a una esfera de la vida constriñe necesariamente el tiempo restante para las otras. En este sentido, un mayor número de horas trabajando en el mercado laboral implicará una reducción del tiempo disponible para el cuidado de los hijos (Presser, 1994) y las tareas del hogar (Coverman, 1985). A su vez, se postula que la división del trabajo no remunerado está condicionada por las restricciones de tiempo de los miembros del hogar. Así, la contribución de cada individuo no solo se ve determinada por su propia inversión de tiempo en el trabajo remunerado, sino también por el de sus co-residentes. Si tenemos en cuenta esta perspectiva, las madres monoparentales padecen ciertas desventajas respecto a sus homólogas biparentales. En primer lugar, por la falta de una pareja en el hogar con la que distribuir el tiempo de trabajo no remunerado. Por otra, por tener que lidiar con mayores constreñimientos económicos al disponer solamente de su salario, reduciendo la posibilidad de subcontratar servicios de cuidado o asistencia. La monoparentalidad implicaría una reducción del tiempo disponible para la progenitora y un aumento de las demandas para el resto de miembros del hogar.

Por otro lado, la construcción simbólica de roles de género o doing gender, aporta elementos muy significativos para explicar la desigual división del trabajo entre hombres y mujeres (Hochschild y Machung 1989; Eagly, 1983). La interiorización de una serie de normas e ideologías lleva a hombres y mujeres a reproducir determinados patrones asociados con su género. La distribución del trabajo será desigual, incluso en aquellas parejas en los que los dos miembros trabajen, invirtiendo la mujer más tiempo en el cuidado y el trabajo del hogar y el hombre en el trabajo remunerado y el ocio. De hecho, España no se encuentra precisamente entre los países más destacados en el undoing gender, concepto creado para referirse a las interacciones sociales que reducen las diferencias de género (Deutsch, 2007), siendo los hombres con actitudes no tradicionales hacia las tareas domésticas aún claramente minoritarios (Dominguez-Folgueras et al., 2015; Sevilla-Sanz, Giménez-Nadal y Fernández, 2010). 
Las perspectivas teóricas apuntan, pues, a una mayor dedicación de la mujer a trabajo no remunerado cuando convive con su pareja. Pero, ¿qué sucede cuando la figura del padre desaparece del hogar? En el ámbito internacional encontramos diversos estudios que tratan de comprender la lógica del uso del tiempo de las madres monoparentales. Los resultados no son concluyentes. Mientras que para unos autores la monoparentalidad es sinónimo de menor tiempo de las madres con sus hijos (Sandberg y Hofferth, 2001; Thomson, Mclanahan y Curtin, 1992), para otros no existe tal reducción (Kendig y Bianchi, 2008). Mayor consenso genera el impacto de la monoparentalidad en el tiempo dedicado a las tareas del hogar (Sanik y Mauldin, 1986; Brandwein, Brown y Fox, 1974) ya que, en la medida de lo posible, las madres reducen el tiempo dedicado a las tareas del hogar para proteger, precisamente, el tiempo con los hijos (Bianchi, 2000). Responderían, así, a las normas y valores culturales vinculados a la maternidad intensiva (Lee et al., 2014) según las cuales la madre debe dedicar gran cantidad de tiempo a los hijos (Arendell, 2001). En una sociedad, la española, fuertemente marcada por la tradicional división de género (Domínguez-Folgueras, 2015; Esping-Andersen et al., 2013; González, Jurado y Naldini, 2000), los roles de género siguen perpetuando el papel de la "buena madre" (Shirani, Henwood y Coltart, 2012).

\section{OBJetIVO E HIPÓTESIS}

Tal y como acabamos de ver, la literatura sobre monoparentalidad femenina destaca algunas características relacionadas con la ausencia de una pareja en el hogar: una mayor ocupación que las madres biparentales, un mayor tiempo de trabajo -y más precario- y la búsqueda de apoyo en miembros ajenos al núcleo —mayoritariamente abuelas - que, en algunos casos, se traduce en hogares más complejos (Hernández-Monleón, 2016; Treviño, 2011).

En cuanto a la mayor ocupación, si bien era cierta en 2001, en los últimos años, ya sea por la crisis económica o por otras circunstancias, se ha revertido la tendencia. Según el censo de 2011, prácticamente no hay diferencias entre unas y otras: un 59,2 \% de las madres monoparentales que conviven con algún hijo menor de edad se encuentran ocupadas, mientras que en las biparentales este porcentaje es solamente un punto y medio inferior, un $57,7 \%{ }^{3}$

A pesar de esta tendencia, y tal y como veremos más adelante, el tiempo empleado en el trabajo remunerado por parte de las madres monoparentales sigue siendo significativamente mayor que el tiempo empleado por las madres biparentales una hora diaria, aproximadamente-, así como la complejidad de los núcleos es superior en el primer caso que en el segundo. ${ }^{4}$
A partir de esta premisa, el primer objetivo que nos planteamos es valorar si este mayor tiempo empleado por la madre en el trabajo remunerado tiene su contrapartida en el trabajo no remunerado, distinguiendo entre trabajo de cuidado y trabajo doméstico. En este sentido nos planteamos dos hipótesis:

(H1) Siguiendo a los autores que consideran que el tiempo empleado en el cuidado de los hijos es altamente valorado, dado el auge de la parentalidad intensiva (Lee et al., 2014; Sayer y Gornick, 2012; Arendell, 2001), consideramos que no habrá reducción alguna en este tiempo, de modo que las madres biparentales y monoparentales dedicarán el mismo tiempo a sus hijos.

(H2) En contrapartida, y como el trabajo doméstico es necesario pero no comporta ningún valor añadido, es ahí donde las madres monoparentales se van a diferenciar de las biparentales, empleando menos tiempo en tareas del hogar. Si esta hipótesis se cumple, nos planteamos como objetivo evaluar qué actividades domésticas son aquellas que las madres monoparentales ahorran tiempo.

$Y$, ¿qué sucede con el resto de miembros? En la literatura existe cierta discrepancia sobre el tiempo que emplean los hijos monoparentales y biparentales en trabajo doméstico. ${ }^{5}$ Tal y como hemos visto, algunos estudios de la década de los noventa llevados a cabo en Estados Unidos indicaban que los hijos de madres monoparentales tenían una mayor aportación que los hijos de madres biparentales (Gager, Cooney y Call, 1999; Demo y Acock, 1993; Goldscheider y Waite, 1991). Por otra parte, estudios más recientes matizan estos resultados en función del tipo de custodia de los padres (Gager, Sanchez y Demaris, 2009). ¿Cuál es la lógica que se cumple actualmente en España? En nuestro caso no podemos valorar la cuestión de la custodia, pero, a pesar de ello, la hipótesis que planteamos es que la diferencia será escasa. Así:

(H3) El tiempo que los hijos dedican al trabajo no remunerado es muy escaso y no se observarán diferencias significativas entre los hijos de madres monoparentales y los hijos de madres biparentales.

En cuanto a los miembros del hogar ajenos al núcleo, debemos separar dos cuestiones: cuántos son y cuál es la aportación de cada uno de ellos al trabajo no remunerado. Es decir, por el simple hecho de que en los hogares monoparentales existe un mayor número de miembros ajenos al núcleo, su aportación global al trabajo no remunerado ya debería ser mayor que en los hogares biparentales. Ahora bien, si, tal y como apunta la literatura, la convivencia con otros miembros de las madres monoparentales es una estrategia de conciliación (Treviño, 2011), deberíamos observar que, de modo individual, cada uno de los miembros ajenos al núcleo monoparental debería hacer una mayor con- 
tribución que los miembros ajenos a los núcleos biparentales. Y así lo planteamos, tanto en tiempo de cuidado como en tiempo de trabajo doméstico:

(H4) A pesar de que consideramos que la madre monoparental dedicará el mismo tiempo de cuidado que la madre biparental $(\mathrm{H} 1)$, las personas ajenas a los núcleos monoparentales van a dedicar más tiempo al cuidado que sus iguales en los núcleos biparentales, las cuales cuentan con el tiempo empleado por el padre residente.

(H5) En el tiempo empleado en trabajo domésti$\mathrm{co}$, y previendo que la madre monoparental le dedica menos tiempo que la madre biparental $(\mathrm{H} 2)$, los miembros ajenos al núcleo monoparental también van a aportar un mayor tiempo que los miembros ajenos residentes en los núcleos biparentales.

\section{Metodología Y FUentes de datos}

La base de datos utilizada para el análisis ha sido la Encuesta de Empleo del Tiempo 2009-2010 del Instituto Nacional de Estadística. Entre otra información, la encuesta dispone de un diario que recoge, para un día concreto de la semana y en intervalos de diez minutos, cuál es la actividad principal desarrollada por todos los miembros del hogar de diez y más años. Precisamente es el diario lo que permite que la información recogida sea lo más precisa posible, haciendo de las encuestas de uso del tiempo un instrumento válido y fiable. ${ }^{6}$

Siguiendo la definición y el objetivo del análisis, se han seleccionado los hogares en los que convive una mujer ocupada con algún hijo menor de 18 años. Por las dificultades de clasificación, han sido excluidos aquellos hogares en los que conviven núcleos monoparentales y biparentales, ambos con hijos menores. También se han excluido los hogares que dicen contar con un adulto en situación de dependencia, ${ }^{7}$ ya que esto podría comportar un sesgo importante, sobre todo en el análisis del tiempo correspondiente a los miembros ajenos al núcleo. ${ }^{8}$ La muestra final es de 1.660 hogares: 182 monoparentales y 1.478 biparentales.

Para la mayoría de los análisis realizados se distingue entre tiempo de cuidado y tiempo doméstico. El primero se circunscribe al cuidado de menores de edad —cuidados físicos y vigilancia, leer, jugar, conversar, acompañamiento, etc.-; mientras que el tiempo doméstico es aquel empleado en tareas del hogar —cocina, mantenimiento del hogar, confección, jardinería, reparaciones, compras y gestión-. Cuando se analicen conjuntamente los dos tiempos, hablaremos del tiempo empleado en trabajo no remunerado.

En primer lugar, y para contrastar las hipótesis 1 y 2 , se han desarrollado modelos para el tiempo de cuidado y para el tiempo doméstico, siendo la variable dependiente el tiempo empleado por las madres. Desde un punto de vista metodológico, para evaluar las diferencias de tiempo entre hogares monoparentales y biparentales se han realizado regresiones lineales de mínimos cuadrados ordinarios, utilizando como categoría de referencia los hogares biparentales. ${ }^{9}$

En una primera aproximación se han generado modelos con el tipo de núcleo —monoparental o biparental- como variable dependiente y controlando por el día de la semana, siendo su inclusión necesaria debido a que en la muestra están sobre-representados los fines de semana (modelo 1 ).

A continuación, y para profundizar en las diferencias, se han desarrollado modelos que incorporan las variables que la literatura señala como relevantes: edad de la madre, nivel de estudios, ingresos, presencia de servicio doméstico y país de nacimiento. En el análisis del tiempo de cuidado se ha introducido, además, el número de hijos menores de 18 años, así como la edad del hijo pequeño. Con el fin de obtener un modelo más parsimonioso, se ha procedido a eliminar del modelo completo, una a una, las variables no significativas con una menor aportación, eliminándose todas las variables con un nivel de significación mayor de 0,10 (modelo 2). Las únicas variables que se han conservado, independientemente de su significación, han sido: la variable dependiente, tipo de núcleo —-monoparental o biparental- y la variable de control, día de la semana -laborable o fin de semana-. El resultado final han sido dos modelos, uno para el tiempo de cuidado y otro para el tiempo doméstico, que contienen únicamente aquellas variables significativas, siendo de especial importancia si en este modelo final el tipo de núcleo es o no significativo. ${ }^{10}$

Se ha desestimado la incorporación a los modelos de las variables asociadas al horario laboral, como puede ser el tipo de jornada, el horario, el número de horas trabajadas, etc. Se ha considerado que la incorporación de estas variables comportaría una pérdida de validez del modelo: si las madres monoparentales están ocupadas un mayor número de horas que las biparentales, esto forma parte precisamente de los problemas que puedan tener de conciliación, de modo que su inclusión en el modelo podría comportar una tautología. En otras palabras: nos interesa conocer si las madres monoparentales ocupadas tienen más problemas de conciliación, y no si a igualdad de horas trabajadas tienen más problemas de conciliación. A pesar de ello, y cuando los resultados han apuntado a estos problemas, sí que se han generado modelos que han incluido el número de horas trabajadas en el mercado laboral. Unos modelos que deberían confirmar o descartar si las diferencias pueden ser explicadas, precisamente, por la diferencia en el número de horas trabajadas. Un razonamiento parecido se ha llevado a cabo con la posible con- 
vivencia con otros miembros, desestimándose esta variable de los modelos: interesa ver si las madres monoparentales dedican más o menos tiempo a sus hijos, y no si este mayor o menor tiempo viene condicionado por la presencia de otros miembros.

Se ha procedido de manera similar en el análisis de los tiempos de los descendientes, así como de los miembros del hogar ajenos al núcleo. Desde un punto de vista metodológico, cabe destacar que, en algunos casos, los hijos y los miembros ajenos al núcleo no han rellenado el diario de actividades. En estos casos disponemos solamente de información sobre el sexo, la edad y la relación con la actividad, variables que se han utilizado para valorar las diferencias entre aquellos que han respondido el diario y los que no.

En el caso de los hijos, en los 1.660 hogares analizados encontramos un total de 2.850 hijos, de los cuales 1.401 tenían diez años o más y deberían haber rellenado el diario. De estos, 948 lo han rellenado y 453 no. Se ha verificado que no existían diferencias significativas entre ambos colectivos. Así lo ha mostrado el test de la ji-cuadrado para el sexo ( $p$-valor $=0,990)$ y para la relación con la actividad ( $p$-valor=0,302), mientras que el test de comparación de medias ha hecho lo propio con la edad ( $p$-valor=0,630).

En cuanto a los miembros ajenos al núcleo, solamente están presentes en 113 hogares, con un total de 185 miembros, de los cuales 130 han rellenado el diario y 55 no. En este caso, si bien el test de la ji-cuadrado muestra que tampoco se observan diferencias significativas en cuanto al sexo ( $p$-valor=0,204), ni en cuanto a la relación con la actividad ( $p$-valor $=0,467)$, el test de comparación de medias ha mostrado que, en el caso de la edad, aquellos que no han rellenado el diario de actividades son algo más jóvenes que aquellos que sí lo han hecho ( $p$ valor=0,068). Teniendo en cuenta que se buscan las diferencias entre hogares monoparentales y biparentales, se ha valorado la incidencia de este pequeño sesgo y se ha comprobado que esta diferencia de edad se producía en ambos tipos de hogar, de modo que no debería incidir en los resultados.

Metodológicamente, tanto para los hijos como para las personas ajenas al núcleo, se ha procedido de manera similar a las madres, si bien para los hijos se ha analizado la variable 'tiempo empleado en trabajo no remunerado', y no las variables 'tiempo de cuidado' y 'tiempo doméstico'. En cada uno de los casos, se han generado dos modelos. En el primero solamente están presentes las variables dependientes y de control - tipo de núcleo y día de la semana, respectivamente- (modelo 1); mientras que en el segundo están presentes, además de estas dos, todas las variables significativas (modelo 2). Las diferencias más importantes con los modelos generados para la madre corresponden a las variables 'relación con la actividad' y 'nivel de ingresos'. La relación con la actividad, que no tenía sentido incorporarla para las madres, ahora sí que ha sido utilizada; mientras que el nivel de ingresos, al tener solamente sentido en las personas ocupadas, se ha desestimado en los modelos generados para los hijos y para los miembros ajenos al núcleo.

\section{Características de los núcleos MONOPARENTALES Y BIPARENTALES}

Debe recordarse, en primer lugar, que solamente se analizan madres ocupadas que conviven con algún hijo menor de 18 años. Según la Encuesta de Empleo del Tiempo de 2009-2010, la ocupación de las madres monoparentales es de un $64,7 \%$, mientras que la de las biparentales es del 61,0\% ( $p$-valor=0,235). ${ }^{11}$ En cambio, y aunque no afecta a los resultados de nuestro estudio, las diferencias en el resto de categorías de la relación con la actividad son mucho mayores: entre las monoparentales no ocupadas, un $76 \%$ se declara en paro, mientras que entre las biparentales no ocupadas la opción mayoritaria es declarar que están realizando tareas del hogar; un $60 \%$ así lo manifiesta.

De las características socioeconómicas que se desprenden de la Encuesta de Empleo del Tiempo (tabla 1), cabe señalar una mayor precariedad en las madres monoparentales que en las biparentales, en prácticamente todos los indicadores analizados. Destacan, en primer lugar, por un nivel de estudios significativamente menor, por una mayor presencia de madres nacidas en el extranjero y por contar con cierta desventaja en cuanto a los ingresos, agravada por trabajar un mayor número de horas. Además, y especialmente relevante para la conciliación, es menor la presencia de servicio doméstico en los hogares monoparentales.

Atendiendo a las características demográficas, no se observan diferencias significativas en la edad media de las madres monoparentales y biparentales, aunque sí en el número de hijos menores de 18 años y, sobre todo, en la edad de los mismos: el hijo pequeño de las madres monoparentales es unos dos años mayor que el de las biparentales.

Otra cuestión, que más adelante retomaremos, es la presencia de miembros del hogar ajenos al núcleo. La proporción de madres que conviven con otros miembros es significativamente mayor en los hogares monoparentales que en los biparentales, algo que ya nos advierte la literatura (HernándezMonleón, 2016; Treviño, 2011; Tobío y Fernández, 1999). Además, en los hogares monoparentales los individuos que no forman parte del núcleo son más numerosos: su tamaño medio es de 1,85 personas, mientras que en los hogares biparentales es de 1,42 . 
Tabla 1

Características de las madres analizadas.

\begin{tabular}{|c|c|c|c|c|}
\hline & $\begin{array}{l}\text { Muestra } \\
(\mathrm{n}=1.660)\end{array}$ & $\begin{array}{c}\text { Monoparentales } \\
\qquad(n=182)\end{array}$ & $\begin{array}{c}\text { Biparentales } \\
\qquad(n=1.478)\end{array}$ & Significación ${ }^{s}$ \\
\hline \multicolumn{5}{|l|}{ Nivel de estudios } \\
\hline Obligatorios o inferiores & 521 & $41,8 \%$ & $30,2 \%$ & $* * *$ \\
\hline Bachillerato postobligatorio & 395 & $28,8 \%$ & $23,1 \%$ & * \\
\hline Universitarios & 744 & $29,4 \%$ & $46,7 \%$ & $* * *$ \\
\hline \multicolumn{5}{|l|}{ País de nacimiento } \\
\hline España & 1.321 & $70,1 \%$ & $80,7 \%$ & $* * *$ \\
\hline Fuera de España & 339 & $29,9 \%$ & $19,3 \%$ & $* * *$ \\
\hline \multicolumn{5}{|l|}{ Ingresos por trabajo } \\
\hline $600 €$ o menos & 288 & $14,7 \%$ & $17,7 \%$ & NS \\
\hline De 601 a 1.200 & 639 & $47,5 \%$ & $37,4 \%$ & ** \\
\hline De 1.201 a 1.600 & 269 & $9,6 \%$ & $17,0 \%$ & $* *$ \\
\hline Más de $1.600 €$ & 273 & $15,3 \%$ & $16,6 \%$ & NS \\
\hline NS/NC & 191 & $13,0 \%$ & $11,3 \%$ & NS \\
\hline \multicolumn{5}{|l|}{ Horas diarias trabajadas ${ }^{1}$} \\
\hline Media & & 5,83 & 4,87 & $* * *$ \\
\hline \multicolumn{5}{|l|}{ Servicio doméstico en el hogar } \\
\hline No & 1.428 & $90,4 \%$ & $85,5 \%$ & * \\
\hline Sí & 232 & $9,6 \%$ & $14,5 \%$ & * \\
\hline \multicolumn{5}{|l|}{ Edad de la madre } \\
\hline Media & & 39,79 & 39,03 & NS \\
\hline \multicolumn{5}{|l|}{ Número de hijos menores de edad } \\
\hline Media & & 1,40 & 1,55 & $* * *$ \\
\hline \multicolumn{5}{|l|}{ Edad del hijo pequeño } \\
\hline Media & & 9,06 & 6,85 & $* * *$ \\
\hline \multicolumn{5}{|l|}{ Miembros ajenos al núcleo } \\
\hline Núcleo solo & 1.547 & $80,8 \%$ & $94,7 \%$ & $* * *$ \\
\hline Núcleo con otros & 113 & $19,2 \%$ & $5,3 \%$ & *** \\
\hline \multicolumn{5}{|l|}{ El uso del tiempo } \\
\hline Tiempo empleado en cuidado & & $1: 15$ & $1: 53$ & $* * *$ \\
\hline Tiempo empleado en trabajo doméstico & & $2: 57$ & $3: 18$ & $* * *$ \\
\hline
\end{tabular}

1. Las horas trabajadas han sido extraídas del diario de actividades.

2. La significación para las variables categóricas se corresponde con la de los residuos corregidos de la tabla de contingencia; mientras que en las variables numéricas se han calculado a partir del T-test de comparación de dos medias independientes: $0<{ }^{* * *}<0,01<{ }^{* *}<0,05<{ }^{*}<0,10<\mathrm{NS}<1$.

Fuente: Elaboración propia a partir de la Encuesta de Empleo del Tiempo, 2009-2010 (INE).

\section{RESULTADOS}

\section{El tiempo de cuidado y el tiempo doméstico de las madres}

El conjunto de madres ocupadas, ya sean monoparentales o biparentales, dedican diariamente 1 hora y 49 minutos al cuidado y 3 horas y $15 \mathrm{mi}-$ nutos al trabajo doméstico.

Ciñéndonos al trabajo de cuidado, las madres monoparentales dedican a sus hijos 38 minutos me- nos que las madres biparentales (tabla 2, modelo 1). Esta diferencia se reduce completamente y pasa a ser no significativa al introducir las variables que han resultado significativas (tabla 2 , modelo 2 ). Después de testar qué variable ha comportado que no existan diferencias en el tiempo de cuidado entre unas madres y otras, se ha observado que es la edad del hijo pequeño. $Y$ es que, tal y como se ha visto en la tabla 1, los hijos de las madres monoparentales son unos dos años mayores que los hijos de las madres biparentales. Al introducir esta variable la diferencia entre unas y otras pasa a ser no significativa. 
En cuanto al resto de variables, cabe señalar que el número de hijos menores de edad no comporta diferencia alguna, así como tampoco son significativos la presencia de servicio doméstico ni el país de nacimiento de la madre. En cambio, sí que destaca un mayor tiempo de cuidado los días laborables, una mayor dedicación por parte de las madres universitarias, una relación directa entre el tiempo de cuidado y los ingresos, así como una mayor dedicación por parte de las madres de más edad.

En cuanto al tiempo empleado en el trabajo doméstico, vemos que las madres monoparentales dedican a esta labor unos 20 minutos diarios menos que las madres biparentales (tabla 3 , modelo 1). Esta diferencia se incrementa hasta los 28 minutos al introducir las variables de control significativas (tabla 3 , modelo 2 ). ${ }^{12}$

En cuanto a esta variable son significativas el día de la semana, destinándose a trabajo doméstico unos quince minutos más los días laborables que los fines de semana; el nivel de estudios de la madre, siendo las madres universitarias las que menos tiempo le dedican; el nivel de ingresos, de modo que las madres con un menor nivel de ingresos dedican más tiempo a trabajo doméstico; la presencia de servicio doméstico, comportando un ahorro de media hora diaria en este tipo de labores, y la edad de la madre, que mantiene una relación directa con el trabajo doméstico.

Pero, ¿qué dejan de hacer las madres monoparentales en esta media hora?

Las diferencias significativas corresponden a las actividades culinarias -incluye fregar la vajilla-, al mantenimiento del hogar — básicamente, limpieza—, al cuidado de la ropa y a actividades inespecíficas (tabla 4). Dejando al lado estas últimas, la principal reducción en términos absolutos la encontramos en la cocina y en el mantenimiento, actividades en las que las madres monoparentales destinan unos diez minutos menos que las madres biparentales. Ahora bien, si tenemos en cuenta la reducción relativa, debemos apuntar hacia las actividades relacionadas con la ropa, ya que, si bien la diferencia solamente es de seis minutos, la estimación del tiempo medio a esta actividad ya es de por sí muy reducida, de unos veinte minutos. Nuestros resultados van en la línea esperada, ya que la reducción del tiempo invertido en las tareas más rutinarias o arduas, como cocinar o limpiar, ya había sido observada tanto para el conjunto de las mujeres (Bianchi, Robinson y Milkie, 2006; Becker y Moen, 1999) como para las monoparentales (Sanik y Mauldin, 1986).

Tabla 2

Modelos para el tiempo de cuidado para las madres.

\begin{tabular}{|c|c|c|c|c|}
\hline & \multicolumn{2}{|c|}{ Modelo 1} & \multicolumn{2}{|c|}{ Modelo 2} \\
\hline & Coeficiente & Significación & Coeficiente & Significación \\
\hline \multicolumn{5}{|l|}{ Tipo de núcleo } \\
\hline Monoparental & $-0: 38$ & *** & $-0: 02$ & NS \\
\hline Biparental & Ref. & & Ref. & \\
\hline \multicolumn{5}{|l|}{ Día de la semana } \\
\hline Laborable & Ref. & & Ref. & \\
\hline Fin de semana & $-0: 12$ & $* *$ & $-0: 15$ & $* * *$ \\
\hline \multicolumn{5}{|l|}{ Nivel de estudios(a) } \\
\hline No universitarios & & & Ref. & \\
\hline Universitarios & & & $0: 30$ & $* * *$ \\
\hline \multicolumn{5}{|l|}{ Nivel de ingresos ${ }^{(a)}$} \\
\hline$<600 €$ & & & $0: 17$ & * \\
\hline $600-1600$ & & & $-0: 07$ & NS \\
\hline$>1600 €$ & & & $-0: 17$ & * \\
\hline NS/NC & & & Ref. & \\
\hline Edad de la madre & & & 0:02 & *** \\
\hline Edad del hijo pequeño & & & $-0: 14$ & $* * *$ \\
\hline Intersección & $1: 58$ & $* * *$ & $2: 19$ & *** \\
\hline $\mathbf{R}^{2}$ & 0,013 & $* * *$ & 0,358 & *** \\
\hline
\end{tabular}

(a) Tanto en el nivel de estudios como en el nivel de ingresos se han unido las categorías que apenas mostraban diferencias

Significación: $0<* * * 0,01<* *<0,05<{ }^{*}<0,10<$ NS $<1$.

Fuente: Elaboración propia a partir de la Encuesta de Empleo del Tiempo, 2009-2010 (INE). 
Tabla 3

Modelos para el tiempo dedicado a trabajo doméstico por parte de las madres.

\begin{tabular}{|c|c|c|c|c|}
\hline & \multicolumn{2}{|c|}{ Modelo 1} & \multicolumn{2}{|c|}{ Modelo 2} \\
\hline & Coeficiente & Significación & Coeficiente & Significación \\
\hline \multicolumn{5}{|l|}{ Tipo de núcleo } \\
\hline Monoparental & $-0: 21$ & ** & $-0: 28$ & $* * *$ \\
\hline Biparental & Ref. & & Ref. & \\
\hline \multicolumn{5}{|l|}{ Día de la semana } \\
\hline Laborable & Ref. & & Ref. & \\
\hline Fin de semana & $0: 15$ & ** & $0: 17$ & $* * *$ \\
\hline \multicolumn{5}{|l|}{ Nivel de estudios ${ }^{(a)}$} \\
\hline No universitarios & & & Ref. & \\
\hline Universitarios & & & $-0: 14$ & ** \\
\hline \multicolumn{5}{|l|}{ Nivel de ingresos ${ }^{(a)}$} \\
\hline$<600 €$ & & & $0: 48$ & $* * *$ \\
\hline $600-1600$ & & & 0:00 & NS \\
\hline$>1600 €$ & & & $-0: 16$ & NS \\
\hline $\mathrm{NS} / \mathrm{NC}$ & & & Ref. & \\
\hline \multicolumn{5}{|c|}{ Servicio doméstico en el hogar } \\
\hline No & & & Ref. & \\
\hline Sí & & & $-0: 28$ & $* * *$ \\
\hline Edad de la madre & & & $0: 05$ & $* * *$ \\
\hline Intersección & $3: 11$ & $* * *$ & $0: 17$ & NS \\
\hline $\mathbf{R}^{2}$ & 0,006 & $* * *$ & 0,089 & $* * *$ \\
\hline
\end{tabular}

(a) Tanto en el nivel de estudios como en el nivel de ingresos se han unido las categorías que apenas mostraban diferencias Significación: $0<* * * 0,01<* * 0,05<*<0,10<$ NS $<1$.

Fuente: Elaboración propia a partir de la Encuesta de Empleo del Tiempo, 2009-2010 (INE).

Tabla 4

Modelos para cada una de las actividades domésticas. Variables de control: día de la semana, nivel de estudios, nivel de ingresos, servicio doméstico y edad de la madre

\begin{tabular}{|c|c|c|c|}
\hline & Estimación media & $\begin{array}{c}\text { Monoparentales- } \\
\text { biparentales }^{(a)}\end{array}$ & Significación \\
\hline Actividades culinarias & $1: 14$ & $-0: 09$ & ** \\
\hline Mantenimiento del hogar & $0: 45$ & $-0: 10$ & ** \\
\hline Confección y cuidado de ropa & $0: 20$ & $-0: 06$ & ** \\
\hline Compras & $0: 38$ & 0:01 & NS \\
\hline Jardinería & $0: 03$ & $0: 02$ & NS \\
\hline Reparaciones & 0:02 & $-0: 00$ & NS \\
\hline Gestiones & 0:00 & $0: 00$ & NS \\
\hline Actividades inespecíficas & $0: 13$ & $-0: 06$ & * \\
\hline Total trabajo doméstico & $3: 15$ & $-0: 28$ & $* * *$ \\
\hline
\end{tabular}

(a) Aunque aquí se ha expresado como una diferencia, se trata del coeficiente para las madres monoparentales, siendo las madres biparentales la categoría de referencia.

Significación: $0<{ }^{* *}<0,001<* * 0,050<*<0,100<$ NS $<1$.

Fuente: Elaboración propia a partir de la Encuesta de Empleo del Tiempo, 2009-2010 (INE). 


\section{El tiempo de cuidado y el tiempo doméstico de los hijos e hijas}

Una breve pincelada sobre las principales características de la muestra de hijos e hijas de diez y más años evidencia una edad media relativamente joven, 15,07 años, con lo cual no es de extrañar que la inmensa mayoría declare estar estudiando (tabla 5). Del mismo modo, no sorprende tampoco que, preguntados por el nivel de estudios, un $84,3 \%$ declare haber terminado, como mucho, los estudios obligatorios. ${ }^{13}$

En su conjunto, los hijos e hijas dedican al trabajo no remunerado, que incluye cuidado y tiempo doméstico, 52 minutos diarios.

Los resultados muestran que no hay diferencias significativas entre los descendientes de madres monoparentales y biparentales. Pocas variables muestran algún tipo de relación en el tiempo que estos dedican a trabajo no remunerado, siendo siempre el sentido de la relación el esperado (tabla 5). Así, el tiempo de trabajo no remunerado es 27 minutos mayor entre las hijas que entre los hijos, observándose además una mayor dedicación entre los que no se encuentran insertados en el mercado laboral, así como entro los de más edad.

\section{El tiempo de cuidado y el tiempo doméstico de los miembros del hogar ajenos al núcleo}

Cabe destacar, en primer lugar, que la mayor presencia de otros miembros en los hogares monoparen- tales que en los hogares biparentales se traduce tanto en una mayor prevalencia como en un tamaño medio de estos otros miembros más elevado. Tal y como muestra la tabla 1, la prevalencia de otros miembros es del 19,2\% en los hogares monoparentales y del 5,3 $\%$ en los hogares biparentales. En cuanto al tamaño medio, en los hogares biparentales con otros miembros observamos, de media, 1,42 miembros, cifra algo inferior al de los hogares monoparentales, 1,85.

En la muestra disponible, la mayor parte de las personas ajenas al núcleo son alguno de los progenitores de la madre (o del padre en los hogares biparentales), representando algo más del $60 \%$ del total. En segundo lugar, se encuentran los hermanos o hermanas, que representan algo más del $20 \%$, repartiéndose el resto en otras categorías.

Como se ha descrito en la metodología y puede verse en las tablas 6 y 7 , la muestra de personas ajenas al núcleo es muy reducida, de 130 personas, mayoritariamente mujeres, de 59 años de edad media y que se encuentran desocupadas, ya sea en paro o jubiladas.

Estas personas que no forman parte del núcleo dedican diariamente 8 minutos a cuidado y unas tres horas a trabajo doméstico (2:59 horas). Vemos, pues, que, sobre todo en cuanto al trabajo doméstico, se trata de cifras nada desdeñables y que, acompañadas del hecho de una mayor presencia - ya sea en términos de prevalencia o de tamaño medio- en los hogares monoparentales, los indicios que apuntan a un soporte importante para la conciliación son significativos.

Tabla 5

Características de la muestra de hijos e hijas y modelos para el tiempo dedicado al trabajo no remunerado

\begin{tabular}{|c|c|c|c|c|c|}
\hline & \multirow{2}{*}{$\begin{array}{l}\text { Muestra } \\
(n=948)\end{array}$} & \multicolumn{2}{|c|}{ Modelo 1} & \multicolumn{2}{|c|}{ Modelo 2} \\
\hline & & Coeficiente & Significación & Coeficiente & Significación \\
\hline \multicolumn{6}{|l|}{ Tipo de núcleo } \\
\hline Monoparental & $16,6 \%$ & 0:00 & NS & $-0: 06$ & NS \\
\hline Biparental & $86,4 \%$ & Ref. & & Ref. & \\
\hline \multicolumn{6}{|l|}{ Día de la semana } \\
\hline Laborable & $44,1 \%$ & Ref. & & Ref. & \\
\hline Fin de semana & $55,9 \%$ & 0:06 & NS & $0: 07$ & NS \\
\hline \multicolumn{6}{|c|}{ Relación con la actividad } \\
\hline Ocupados & $5,2 \%$ & & & Ref. & \\
\hline Estudiantes & $91,7 \%$ & & & $0: 30$ & $* * *$ \\
\hline Otros & $3,1 \%$ & & & $1: 11$ & $* * *$ \\
\hline \multicolumn{6}{|l|}{ Sexo } \\
\hline Hombre & $53,2 \%$ & & & $-0: 27$ & $* * *$ \\
\hline Mujer & $46,8 \%$ & & & Ref. & \\
\hline Edad del hijo & 15,07 & & & $0: 03$ & $* * *$ \\
\hline Intersección & & $0: 49$ & $* * *$ & $-0: 13$ & NS \\
\hline $\mathbf{R}^{2}$ & & 0,002 & NS & 0,079 & $* * *$ \\
\hline
\end{tabular}

Significación: $0<{ }^{* *}<0,01<{ }^{* *}<0,05<{ }^{*}<0,10<$ NS $<1$.

Fuente: Elaboración propia a partir de la Encuesta de Empleo del Tiempo, 2009-2010 (INE). 
Pero las hipótesis que planteamos van más allá y se encaminaban hacia una mayor implicación de estas personas en los hogares monoparentales que en los biparentales.

En cuanto al tiempo de cuidado, los resultados muestran que hay diferencias significativas entre aquellos que conviven con madres monoparentales y aquellos que lo hacen con biparentales (tabla 6); de modo que las personas que conviven con una madre monoparental dedican al trabajo de cuidado unos diecisiete minutos más que las personas que conviven con una madre biparental. Así, observamos que no solamente las madres monoparentales conviven con más miembros, sino que estos, de modo individual, destinan más tiempo al cuidado que los miembros que conviven con las madres biparentales.

En cuanto al resto de variables cabe destacar, en primer lugar, que, tal y como sucede con el tiempo de cuidado de la madre, a mayor edad del hijo menor es la aportación llevada a cabo por los miembros ajenos al núcleo. Además, las mujeres —normalmente abuelas o tías- destinan al cuidado 17 minutos más que los hombres; un tiempo que es mayor los fines de semana y para las que tienen un menor nivel de estudios.

Respecto del tiempo empleado en el trabajo doméstico (tabla 7), los resultados muestran que no existen diferencias significativas entre las personas que conviven con una madre monoparental y las personas que conviven con una madre biparental. Después de controlar por las variables significativas, las diferencias que se observan entre ambas son de unos veinte minutos a favor de las personas que conviven con madres monoparentales, de modo que los indicios apuntan a que estas prestan más ayuda en términos de tiempo, aunque siendo las diferencias estadísticamente no significativas. Es decir, no podemos asegurar que, de media, las personas que acompañan los núcleos monoparentales sean distintas de las personas que acompañan los núcleos biparentales. Ahora bien, no podemos olvidar que la presencia de estas personas es cuatro veces superior en los núcleos monoparentales que en los biparentales, que estas son más en número y que cada una de estas personas dedica unas tres horas diarias al tiempo doméstico.

En cuanto al resto de variables, es destacable que, en los pocos hogares con servicio doméstico, la aportación de los otros miembros es claramente inferior; que las mujeres aportan a trabajo doméstico un tiempo significativamente mayor que los hombres; que a mayor edad más tiempo a trabajo doméstico, y que encontrarse en el mercado laboral es un factor que inhibe la ayuda a las tareas domésticas.

Tabla 6

Características de la muestra de miembros del hogar ajenos al núcleo, y modelos para el tiempo de cuidado.

\begin{tabular}{|c|c|c|c|c|c|}
\hline & \multirow{2}{*}{$\begin{array}{c}\text { Muestra } \\
(n=130)\end{array}$} & \multicolumn{2}{|c|}{ Modelo 1} & \multicolumn{2}{|c|}{ Modelo 2} \\
\hline & & Coeficiente & Significación & Coeficiente & Significación \\
\hline \multicolumn{6}{|l|}{ Tipo de núcleo } \\
\hline Monoparental & $46,2 \%$ & $0: 18$ & ** & $0: 17$ & * \\
\hline Biparental & $53,8 \%$ & Ref. & & Ref. & \\
\hline \multicolumn{6}{|l|}{ Día de la semana } \\
\hline Laborable & $36,9 \%$ & Ref. & & Ref. & \\
\hline Fin de semana & $63,1 \%$ & $0: 17$ & * & $0: 15$ & * \\
\hline \multicolumn{6}{|l|}{ Nivel de estudios } \\
\hline Obligatorios & $74,6 \%$ & & & Ref. & \\
\hline Post-obligatorios & $25,4 \%$ & & & $-0: 16$ & * \\
\hline \multicolumn{6}{|l|}{ Sexo } \\
\hline Hombre & $40,8 \%$ & & & $-0: 17$ & ** \\
\hline Mujer & $59,2 \%$ & & & Ref. & \\
\hline Edad del hijo pequeño & 6,36 & & & $-0: 02$ & ** \\
\hline Intersección & & $0: 04$ & NS & $0: 29$ & $* * *$ \\
\hline $\mathbf{R}^{2}$ & & 0,061 & $* *$ & 0,149 & $* * \star$ \\
\hline
\end{tabular}

Los asteriscos significan: $0<{ }^{* * *}<0,01<{ }^{* *}<0,05<*<0,10<\mathrm{NS}<1$

Fuente: Elaboración propia a partir de la Encuesta de Empleo del Tiempo, 2009-2010 (INE). 
Tabla 7

Características de la muestra de miembros del hogar ajenos al núcleo y modelos para el tiempo de trabajo doméstico

\begin{tabular}{|c|c|c|c|c|c|}
\hline & \multirow{2}{*}{$\begin{array}{c}\text { Muestra } \\
(n=130)\end{array}$} & \multicolumn{2}{|c|}{ Modelo 1} & \multicolumn{2}{|c|}{ Modelo 2} \\
\hline & & Coeficiente & Significación & Coeficiente & Significación \\
\hline \multicolumn{6}{|l|}{ Tipo de núcleo } \\
\hline Monoparental & $46,2 \%$ & $-0: 02$ & NS & $0: 21$ & NS \\
\hline Biparental & $53,8 \%$ & Ref. & & Ref. & \\
\hline \multicolumn{6}{|l|}{ Día de la semana } \\
\hline Laborable & $36,9 \%$ & Ref. & & Ref. & \\
\hline Fin de semana & $63,1 \%$ & $0: 42$ & NS & $-0: 20$ & NS \\
\hline \multicolumn{6}{|c|}{ Servicio doméstico en el hogar } \\
\hline No & $93,8 \%$ & & & Ref. & \\
\hline Sí & $6,2 \%$ & & & $-2: 12$ & $* *$ \\
\hline \multicolumn{6}{|c|}{ Relación con la actividad } \\
\hline Ocupado & $30,8 \%$ & & & $-1: 22$ & ** \\
\hline Parado/jubilado & $69,2 \%$ & & & Ref. & \\
\hline Edad del miembro & 59,13 & & & 0:03 & ** \\
\hline \multicolumn{6}{|l|}{ Sexo } \\
\hline Hombre & $40,8 \%$ & & & $-1: 24$ & $* * *$ \\
\hline Mujer & $59,2 \%$ & & & Ref. & \\
\hline Intersección & & $2: 41$ & $* * *$ & 2:01 & * \\
\hline $\mathbf{R}^{2}$ & & 0,013 & NS & 0,326 & $* * *$ \\
\hline
\end{tabular}

Fuente: Elaboración propia a partir de la Encuesta de Empleo del Tiempo, 2009-2010 (INE).

\section{CONCLUSIONES}

La madre trabajadora se encuentra ante una encrucijada. Por un lado, responder a las presiones sociales vinculadas a los roles de género que hacen de ella la principal responsable del trabajo no remunerado (Sevilla-Sanz, Giménez-Nadal y Fernández, 2010), y, por el otro, enfrentarse a la escasez de tiempo derivada de su participación activa en el mercado de trabajo, con el constreñimiento temporal que eso supone. En este sentido, estudios anteriores han señalado que la incorporación de la mujer al trabajo remunerado no se ha traducido en una reducción del tiempo dedicado a sus hijos (Bianchi, Robinson y Milkie, 2006; Sayer, Gauthier y Furstenberg, 2004; Sandberg y Hofferth, 2001).

Pero, ¿qué sucede con las madres monoparentales? En su caso, las dificultades para la conciliación de la vida familiar y laboral se ven agravadas por la ausencia del hogar de la otra figura parental con la que compartir tanto las expensas económicas como el reparto de las tareas no remuneradas. Así, sin la aportación del padre residente, la madre monoparental intenta zafarse de sus problemas de tiempo, ya sea redistribuyendo su propio tiempo o buscando ayuda entre los miembros del hogar. Los resultados alcanzados en este artículo no permiten afirmar que los hijos de las madres monoparentales tengan una mayor contribución a actividades domésticas que los hijos de madres biparentales (hipótesis 3 ), algo que algunos autores han apuntado para otros países. Tampoco podemos concluir, aunque los indicios apunten en esta dirección, que los miembros del hogar ajenos al núcleo monoparental dediquen más tiempo a las tareas domésticas que sus homólogos biparentales (hipótesis 5).

En este artículo se visualizan dos estrategias de conciliación.

En primer lugar, se ha comprobado que las madres monoparentales tienen una dedicación al trabajo doméstico distinta a las madres biparentales, confirmándose así una de las hipótesis planteadas: las madres monoparentales dedican menos tiempo a las tareas del hogar que sus homólogas biparentales. Dados los constreñimientos de tiempo, las madres monoparentales destinan un tiempo menor a aquellas actividades domésticas más arduas y que probablemente permiten una mayor flexibilidad: las tareas culinarias, de mantenimiento del hogar y aquellas relacionadas con la ropa. En cambio, no 
se ha observado un menor tiempo destinado a las compras, algo que las nuevas tecnologías facilitarían. Aunque no estamos en condiciones de asegurarlo, es probable que las madres monoparentales recurran más a comidas preparadas, reduzcan los estándares de limpieza y apuesten más por la ropa que no necesita de planchado.

Una de las limitaciones más importantes de este artículo es la imposibilidad de establecer, en términos generales, quién es el principal beneficiario del tiempo empleado en las actividades domésticas. En este sentido, es importante tener en cuenta que las madres biparentales cuentan con un miembro adicional que, sin duda, forma parte de la ecuación, el padre. Hemos comprobado que este destina algo menos de dos horas diarias al trabajo doméstico, un tiempo que no sabemos si es suficiente para atender su propia demanda total ni, mucho menos, su demanda en actividades específicas. Por ejemplo, es probable que parte del tiempo de planchado de la madre biparental se destine a cubrir la demanda generada por su pareja, lo que podría explicar la diferencia con las madres monoparentales.

Por otro lado, el tiempo de cuidado permanece intacto. Aunque los primeros resultados mostraban una menor contribución de las madres monoparentales al cuidado, a igualdad de edad del hijo menor esta diferencia desaparece, dedicando el mismo tiempo las madres monoparentales y biparentales. A nuestro entender, el tiempo con los hijos es un tiempo valorado, precioso, que pasa a priorizarse frente a otros.

Las madres monoparentales siguen así las normas y valores culturales vinculados a la maternidad intensiva (Lee et al., 2014), que enfatiza la necesidad de que la madre pase tiempo con sus hijos. La priorización del tiempo de cuidado por encima de otras actividades matiza la idea propuesta por la perspectiva de la disponibilidad de tiempo, según la cual, dada su mayor contribución de tiempo en el trabajo remunerado, las madres monoparentales deberían dedicar menos tiempo al trabajo no remunerado, tanto en actividades domésticas como de cuidado. Los resultados aquí alcanzados van en la línea ya identificada por otras investigaciones, que apuntan que la monoparentalidad no va ligada a la reducción del tiempo con los hijos (Kendig y Bianchi, 2008), pero si a una disminución en las tareas del hogar (Sanik y Mauldin, 1986). En el caso de España, esta lógica ya había sido identificada en el estudio cualitativo realizado por Hernández-Monleón (2016), en el que se señala que, frente a las exigencias en los estándares laborales y de cuidado, una de las estrategias de las madres monoparentales españolas es la minimización de las tareas domésticas.

En segundo lugar, los resultados confirman que, tal y como han señalado distintas autoras (Hernández-Monleón, 2016; Treviño, 2011), convivir con otros miembros es una estrategia de conciliación importante entre las madres monoparentales españolas. Tres resultados apoyan esta afirmación: (1) El $19,4 \%$ de las madres monoparentales vive con otras personas ajenas al núcleo familiar, situación que solamente se da en un $5 \%$ de las madres biparentales. (2) Las madres monoparentales que conviven con miembros ajenos al núcleo lo hacen con un mayor número de personas que las madres biparentales en la misma situación. (3) El tiempo empleado en el trabajo no remunerado por estas personas ajenas al núcleo es muy importante: tres horas al trabajo doméstico y 18 minutos al cuidado.

En este sentido, la principal aportación de este artículo es que se confirma la hipótesis 4, es decir, de forma individual y en lo que al tiempo de cuidado se refiere, la contribución de estos otros miembros es superior en los hogares monoparentales que en los biparentales. Una posible explicación es que estas personas estén cubriendo parte del tiempo que, en los hogares biparentales, dedica el padre. Una afirmación que debe ponerse en cuarentena, ya que desconocemos, en primer lugar, si existe un padre no residente en los hogares monoparentales y, en segundo lugar, cuál es su contribución al cuidado. En un futuro, para comprender el fenómeno en su totalidad, sería importante contar con investigaciones que recogieran cómo se distribuye el tiempo de cuidado entre todos los miembros de las familias monoparentales, residan o no en el hogar.

Así, una de las limitaciones del presente estudio es el análisis en exclusiva de la aportación de los miembros residentes en el hogar. Bien es sabido que la familia transciende las paredes del hogar, por lo que la ayuda familiar va más allá de los resultados mostrados aquí. El apoyo de la familia, en concreto de los abuelos y, sobre todo, de las abuelas, es especialmente importante en España, donde la solidaridad intergeneracional sigue muy presente (Mestre, Guillén y Caro-Blanco, 2012; Tobío, 2012; Pérez, 2007).

Por otro lado, otra de las limitaciones de este trabajo ha sido la necesidad de tratar los hogares monoparentales como una entidad homogénea, sin existir la posibilidad de analizar los distintos perfiles. Futuras investigaciones deberán esclarecer si, dentro del colectivo de madres monoparentales, existen diferentes dinámicas en lo que al uso del tiempo y a las estrategias de conciliación se refiere.

\section{Agradecimientos}

Este artículo se inscribe en el proyecto Espacios de vida y usos del tiempo en las familias del postdivorcio (Ref. CSO2012-39157) subvencionado por el plan nacional I+D+i del Ministerio de Educación y Ciencia; además de formar parte de la tesis doctoral de Núria García Saladrigas: "El uso del tiempo de los hogares reconstituidos y monoparentales". 


\section{NOTAS}

[1] Las cifras revelan que, en España, el $3 \%$ de los embarazos asistidos y el $9 \%$ de las adopciones internacionales fueron por parte de madres solas en el periodo 2000-2004 (González et al., 2008).

[2] A pesar de que existe otra teoría, la del intercambio económico, esta se centra en la negociación entre los dos miembros de la pareja, una situación que no es propia de las mujeres monoparentales y que difícilmente se puede extender al intercambio con otros miembros del hogar.

[3] Estos valores han sido hallados analizando los microdatos del censo de 2011; en concreto, se ha analizado una muestra de 1.602.504 hogares, que contenían 59.581 madres monoparentales con algún hijo menor de edad y 373.423 madres biparentales en la misma situación.

[4] Ambas constataciones provienen de sendos análisis previos que se han llevado a cabo con la Encuesta de Empleo del Tiempo, 2009-2010.

[5] En este caso no tiene demasiado sentido separar el tiempo de cuidado del tiempo doméstico, en tanto que el trabajo de cuidado solamente lo pueden realizar los hijos que tienen hermanos y el tiempo empleado en esta actividad será muy escaso.

[6] Para más información sobre las ventajas e inconvenientes de la EET, véase García-Saladrigas (2018).

[7] El cuestionario define esta situación como la de aquellas personas que, habiendo perdido autonomía física, mental, intelectual o sensorial, precisan de la atención de una o varias personas para realizar actividades básicas de la vida diaria.

[8] Al ser la unidad de análisis el hogar, los miembros ajenos al núcleo serán todas las personas que convivan

\section{RefERENCIAS BiBLIOGRÁfICAS}

Ajenjo, M. y J. García-Román. 2011. "El tiempo productivo, reproductivo y de ocio en las parejas de doble ingreso". Papers. Revista de Sociologia 96:985-1006.

Ajenjo, M. y J. García-Román. 2014. "La transmissió intergeneracional de rols de gènere a la llar". Revista Catalana de Sociologia 29: 35-47. http://doi. org/10.2436/20.3005.01.55

Albertini, M., M. Kohli y C. Vogel. 2007. "Intergenerational transfers of time and money in European families: common patterns- different regimes?" Journal of European Social Policy 17: 319-334. https://doi. org/10.1177/0958928707081068

Almeda, E. y D. Di Nella. 2012. "Análisis de situación de las familias monoparentales en tiempos de crisis a nivel nacional". Comunicación presentada en Jornadas Monomarentales. Mallorca, España.

Almeda, E., D. Di Nella y S. Obiol. 2008. "L'experiència de la monoparentalitat: percepcions, dificultats i demandes". Arxius 19: 19-29.

Arendell, T. 2001. "The new care work of middle class mothers: Managing childrearing, employment, and time". Pp.163-204 en Minding the Time in Family Experience: Emerging Perspectives and Issues, editado por Daly, E. Oxford: Elsevier Science.

Avilés, M. 2015. La monoparentalidad masculina en España. Madrid: CIS. en el mismo hogar y que no formen parte del núcleo principal, independientemente de que tengan o no un vínculo familiar con los miembros del núcleo.

[9] Aunque se han analizado las diferencias de tiempo decimales, para una comprensión más ágil se han convertido todos los coeficientes al formato horas:minutos (hh:mm). Por ejemplo, a un coeficiente de 1,083 horas le corresponde 1:05.

[10] Esta misma lógica se ha utilizado, cuando ha sido posible, en las variables significativas. Así, por ejemplo, el nivel de estudios cuenta inicialmente con tres categorías: obligatorios o inferiores (incluye hasta la Educación Secundaria Obligatoria), Bachillerato postobligatoria (Bachillerato y ciclos formativos de segundo grado) y universitarios (incluye ciclos formativos de tercer grado). Pues bien, en los casos en qué no se observaban diferencias significativas entre algunas categorías, estas se han agrupado.

[11] Tal y como acabamos de ver, según el censo de 2011 , estas cifras son algo inferiores, del $59,2 \%$ entre las madres monoparentales y del $57,7 \%$ entre las biparentales.

[12] Parte de esta diferencia es debida, precisamente, al tiempo de trabajo remunerado. No debemos olvidar que las madres monoparentales dedican prácticamente una hora más al trabajo remunerado que las madres biparentales. Así, controlando en función de este tiempo, las diferencias entre madres monoparentales y biparentales se reduce 13 minutos y deviene no significativa (sig=0,125).

[13] Esta última información no aparece en la tabla 5, al no ser significativa la variable nivel de estudios.

Avilés, M. y A. Sánchez. 2015. "¡Quiero ser madre! Las técnicas de reproducción asistida como vía de acceso a la maternidad en solitario". Cuestiones de género: de la igualdad y la diferencia 10:258-282

Becerril, D. 2004. "Nuevas formas familiares". Portularia $4: 219-230$

Becker, P. E. y P. Moen. 1999. "Scaling back: dual-earner couples' work family strategies". Journal of Marriage and the Family, 61:995-1007.

Bianchi, S. 2011. "Family Change and Time Allocation in American Families". The ANNALS of the American Academy of Political and Social Science 638: 21-44. https://doi. org/10.1177/0002716211413731

Bianchi, S., P. Robinson y M. Milkie. 2006. Changing rhythms of American family life. Nueva York: Russell Sage.

Bianchi, S. 2000. "Maternal employment and time with children: dramatic change or surprising continuity?". Demography 37(4): 401-414. https://doi.org/10.1353/dem.2000.0001

Bianchi, S. y J. Robinson. 1997. "What did you do today? Children's family composition, and the acquisition of social capital". Journal of Marriage and Family 59(2): 332-344. https://doi.org/10.2307/353474

Blair, S. 1992. "The sex-typing of children's household labor: Parental influence on daughters and sons housework". Youth and Society 24: 178-203. 
Brandwein, A., A. Brown y E. Fox. 1974. "Women and Children Last: The Social Situation of Divorced Mothers and Their Families". Journal of Marriage and Family 36(3): 498-514.https://doi.org/10.2307/350721

Brullet, C., M. Marí-Klose, P. Marí-Klose y L. Maranzana. 2011. "Geografías de la paternidad no residente: ¿ausente o vinculado?". Documents d'Anàlisi Geogràfica 57: 83-103.

Castro, T. 2015. "Nuevas familias para un nuevo siglo". Pp. 302-313 en España 2015. Situación social, editado por C. Torres Albero. Madrid: CIS.

Coser, L. 1974. Greedy institutions: Patterns of undivided commitment. New York: Free Press.

Coverman, S. 1985. "Expanding husbands' participation in domestic labor". Sociological Quarterly 26: 81-97.

Demo, D. y A. Acock 1993. "Family diversity and the division of domestic labor: How much have things really changed?". Family Relations 42(3): 323-331.

Deutsch, F. 2007. "Undoing Gender". Gender \& Society 21: 106-127. https://doi.org/10.1177/0891243206293577

Domínguez-Folgueras, M. 2015. "Parentalidad y división del trabajo doméstico en España, 2002-2010". Revista Española de Investigaciones Sociológicas 149: 45-64. https://doi.org/10.5477/cis/reis.149.45

Domínguez-Folgueras, M., T. Jurado, C. Botía y P. Amigot. 2015. 'The house belongs to both': undoing the gendered division of housework". Community, Work \& Family 20 (4): 424-443. https://doi.org/10.1080/13668803. 2016.1192525

Domínguez-Folgueras, M. 2011. Diez años de cambios en las parejas españolas (1995-2006). Madrid: CIS.

Durán, A. 1986. La jornada interminable. Barcelona: Icaria.

Eagly, A. 1983. "Gender and social influence: A social psychological analysis". American Psychologist 38: 971-981. https://doi.org/10.1037/0003-066X.38.9.971

England, P. 2010. "The Gender Revolution". Gender and Society 24(2): 149-166.

Esping-Andersen, G. 2009. The Incomplete Revolution. Cambridge: Polity Press.

Esping-Andersen, G., D. Boertien, J. Bonke, y P. Gracia. 2013. "Couple specialization in multiple equilibria". European Sociological Review 29: 1280-1294.

Evertsson, M. 2006. "The reproduction of gender: housework and attitudes towards gender equality in the home among Swedish boys and girls". The British Journal of Sociology 57: 416-436.

Fernández, J. y C. Tobío. 1998. "Las familias monoparentales en España". Revista Española de Investigaciones Sociológicas 83: 51-85.

Gager, T., T. Cooney y T. Call. 1999. "The Effects of Family Characteristics and Time Use on Teenagers". Journal of Marriage and Family 61: 982-994.

Gager, T., L. Sanchez y A. Demaris. 2009. "Whose time is it? The Effect of Employment and Work/Family Stress on Children's Housework". Journal of Family Issues 30: 1.459-1.485.

García-Román, J. 2012. El uso del tiempo en las parejas de doble ingreso. Tesis Doctoral, Departamento de Geografía, Universitat Autònoma de Barcelona.

García-Saladrigas, N. 2018. El uso del tiempo de los hogares reconstituidos y monoparentales. Tesis Doctoral,
Departamento de Geografía, Universitat Autònoma de Barcelona.

Goldscheider, F., E. Bernhardt y T. Lappegard. 2015. "The Gender Revolution: A Framework for Understanding Changing Family and Demographic Behaviour". Population and Development Review 41(2): 207-239.

Goldscheider, F. 2000. "Men, children and the future of the family in the third millennium". Futures 32: 525-538. https://doi.org/10.1016/S0016-3287(00)00005-7

Goldscheider, F. y L. Waite 1991. New families, no families? Berkeley:University Press.

González, M.J. y T. Jurado. 2015. Padres y madres corresponsables. Una utopía real. Madrid: Catarata.

González, M. y T. Jurado. 2009. “¿Cuándo se implican los hombres en las tareas domésticas? Un análisis de la Encuesta de Empleo del Tiempo". Panorama Social 10: 65-81.

González, M., M. Díez, I. Jiménez y B. Morgado. 2008. "Maternidad a solas por elección: Primera aproximación". Anuario de Psicologia 39(1): 119-126.

González, M., I. Jiménez y B. Morgado. 2004. "Los retos de la maternidad en solitario". Revista de Estudios de Juventud 67: 145-163.

González, M., T. Jurado y M. Naldini. 2000. "Introduction: interpreting the transformation of gender inequalities in southern Europe". Pp. 4-34 en Gender Inequalities in Southern Europe: Women, Work and Welfare in the 1990s. London: Frank Cass.

Gracia, P. 2014. "Fathers' Child Care Involvement and Children's Age in Spain: A Time Use Study on Differences by Education and Mothers' Employment". European Sociological Review 30: 137-150. http://doi. org/10.1093/esr/jcu037

Hernández-Monleón, A. 2016. Ni solas, ni en solitario. Condiciones de vida, solidaridad informal y cuidados en la monomarentalidad. Tesis Doctoral, Departament de Sociologia i Antropologia Social, Universitat de Valencia.

Hochschild, R. y Machung, A. 1989. The second shift: working parents and the revolution at home. New York: Viking Press.

Infestas, M. 2015. La corresponsabilidad familiar en el reparto de tareas domésticas en los hogares de doble ingreso. Tesis Doctoral, Departamento de Economía Aplicada III (Política Económica), Universidad Complutense de Madrid.

Izquierdo, M.J. 1998. El malestar en la desigualdad. Madrid: Cátedra.

Jiménez, I. 2003. "Ser madre sin pareja: circunstancias y vivencias de la maternidad en solitario". Portularia: Revista de Trabajo Social 3: 161-178.

Kendig, S. y S. Bianchi. 2008. "Single, cohabitating, and married mothers' time with children". Journal of Marriage and Family 70(5): 1228-1240. https://doi.org/10.1111/ j.1741-3737.2008.00562.x

Lee, E., J. Bristow, C. Faircloth y J. Macvarish. 2014. Parenting Culture Studies. New York: Palgrave Macmillan.

Longfellow, C. 1979. "Divorce in context: Its impact on children" Pp. 287-306 en Divorce and Separation, editado por G. Levinger y C. Moles. New York: Basic Books.

Maganto, J., I. Bartau y J. Etxeberría. 2003. "La participación de los hijos en el trabajo familiar". Revista de Investigación Educativa 21: 249-269. 
Marks, S. 1977. "Multiple roles and role strain: Some notes on human energy, time and commitment". American Sociological Review 42: 921-936

McDonald, P. 2000: "Gender equity, social institutions and the future of fertility". Journal of Population Research 17: 39-62.

Meil, G. 2011. Individualización y solidaridad familiar. Barcelona: Fundación La Caixa.

Meil, G. 2008. Padres e hijos en la España actual. Colección de Estudios Sociales 19. Barcelona: Fundación La Caixa.

Meil, G. 1999. La postmodernización de la familia española. Madrid: Acento.

Mestre, J. M., J. Guillén y F. Caro-Blanco. 2012. "Abuelas cuidadoras en el siglo XXI: recurso de conciliación de la vida social y familiar". Portularia Revista de Trabajo Social 12: 231-238. http://hdl.handle.net/10272/5973

Moreno, S., M. Ajenjo y V. Borràs. 2016. "La masculinización del tiempo dedicado al trabajo doméstico rutinario". Comunicación presentada en el XII Congreso Español de Sociología (FES). Gijón 30 junio-2 julio 2016.

Nazio, T. 2017. "Family structures, institutional clocks and the organization of childcare in Italy". Comunicación presentada en la 39th IATUR Conference, Madrid, 19-22 Julio 2017

Pérez, L. 2007. Las abuelas como recurso de conciliación entre la vida familiar y laboral. Presente y futuro. Madrid: Instituto de la Mujer.

Presser, B. 1994. "Employment schedules among dual-earner spouses and the division of household labor by gender." American Sociological Review 59: 348-364

Raley, S. 2006. "Children's time use: too busy or not busy enough?" Pp. 142-156 en Rhythms of American Family Life, editado por S Bianchi, P. Robinson y M. Milkie. New York: Russell Sage.

Rivas, A. y I. Jociles. 2013. "Las madres solteras por elección: quiénes son y cómo hemos investigado sus proyectos familiares". Pp. 11-38 en La monoparentalidad por elección: el proceso de construcción de un modelo familiar. Valencia: Tirant lo Blanch.

Sagastizabal, M., y M. Legarreta. 2016. "La «triple presenciaausencia»: una propuesta para el estudio del trabajo doméstico-familiar, el trabajo remunerado y la participación sociopolítica". Papeles del CEIC 1: 1-29. http:// doi.org/10.1387/pceic.15447
Sandberg, J. y S. Hofferth. 2001. "Changes in children's time with parents: United States, 1981-1997". Demography 38(3): 423-436.

Sanik, M y T. Mauldin 1986. "Single Versus Two Parent Families: A Comparison of Mothers' Time”. Family Relations 35(1): 53-56. https://doi.org/10.2307/584282

Sayer, L., y J. C. Gornick. 2012. "Cross-national variation in the influence of employment hours on child care time". European Sociological Review, 28(4): 421-442. https:// doi.org/10.1093/esr/jcr008

Sayer, L., H. Gauthier, y F. Furstenberg. 2004. "Educational Differences in Parents' Time with Children: Cross-National Variations". Journal of Marriage and Family 66: 1152-1169.

Seiz, M. 2016. "The transition to parenthood in Spain: adaptations to ideals". Pp. 196-218 en Coupels' Transitions to Parenthood. Analysing Gender and Work in Europe, editado por D. Grunow y M. Evertsson. Cheltenham: Edward Elgar Publishing.

Sevilla-Sanz, A., I. Giménez-Nadal y C. Fernández. (2010). "Gender Roles and the Division of Unpaid Work in Spanish Households". Feminist Economics 16(4): 137184. https://doi.org/10.1080/13545701.2010.531197

Shirani, J., L. Henwood y C. Coltart, C. 2012. "Meeting the Challenges of Intensive Parenting Culture: Gender, Risk Management and the Moral Parent". Sociology 46: 25-40. https://doi.org/10.1177/0038038511416169

Solsona, M., C. Brullet y J. Spijker. 2014. "Coparentalitat i custòdia compartida a Catalunya". Documents d'Anàlisi Geografica 60: 387-415.

Tobío, C. 2012. "Reciprocity and solidarity in intergenerational relationships : Spain, France and Norway in comparative perspective". Papers 97(4): 849-874.

Tobío, C. y J. Fernández. 1999. "Monoparentalidad, trabajo y familia". Revista Internacional de Sociología 22: 67-97.

Thomson, E., S. Mclanahan y B. Curtin. 1992. "Family Structure, Gender and Parental Socialization". Journal of Marriage and Family 54(2): 368-378.

Treviño, R., J. Gumà e I. Permanyer. 2013. "Las parejas de familias reconstituidas Una caracterización desde la perspectiva de género". Papers de Demografia 149: 1-41.

Treviño, R. 2011. La monoparentalidad en la encrucijada. Madrid: Editorial Académica Española.

MARC AJENJO COSP es Licenciado en Sociología y doctor en Geografía Humana (especialidad Demografía). Ha colaborado en distintos proyectos I+D relacionados con el uso del tiempo y ha dirigido el proyecto El reparto del tiempo y el uso del espacio en las parejas biactivas. La situación española en el contexto Europeo (Ref. CSO2009-08273). Actualmente, imparte docencia en el Departamento de Sociología de la Universitat Autònoma de Barcelona y es investigador asociado al Centre d'Estudis Demogràfics.

NÚRIA GARCÍA SALADRIGAS es Licenciada en Sociología por la Universidad de Barcelona, Máster en Estudios Territoriales y de la Población por la Universidad Autónoma de Barcelona y doctora en Demografía por la Universidad Autónoma de Barcelona. Su tesis titulada "El uso del tiempo de los hogares reconstituidos y monoparentales" fue dirigida por el Dr. Marc Ajenjo y la Dra. Montse Solsona. 